\title{
THE (2,3)-GENERATION OF THE SPECIAL LINEAR GROUPS OVER FINITE FIELDS
}

\author{
MARCO ANTONIO PELLEGRINI
}

\begin{abstract}
We complete the classification of the finite special linear groups $\mathrm{SL}_{n}(q)$ which are $(2,3)$-generated, i.e., which are generated by an involution and an element of order 3 . This also gives the classification of the finite simple groups $\operatorname{PSL}_{n}(q)$ which are $(2,3)$-generated.
\end{abstract}

\section{INTRODUCTION}

It is a well known fact that every finite simple group can be generated by a pair of suitable elements: for alternating groups this is a classical result of Miller [11, for groups of Lie type it is due to Steinberg [17 and for sporadic groups it was proved by Aschbacher and Guralnick [1. A more difficult problem is to find for a finite nonabelian (quasi)simple group $G$ the minimum prime $r$, if it exists, such that $G$ is $(2, r)$-generated, i.e. such that $G$ can be generated by two elements of respective orders 2 and $r$. We denote such minimum prime $r$ by $\varpi(G)$ (setting $\varpi(G)=\infty$ if $G$ is not $(2, r)$-generated for any prime $r)$. Since groups generated by two involutions are dihedral, we must have $\varpi(G) \geq 3$.

Miller himself proved that $\varpi(\operatorname{Alt}(n))=3$ if $n=5$ or $n \geq 9$, while it is easy to verify that $\varpi(\operatorname{Alt}(n))=5$ if $n=6,7,8$. The special linear groups were firstly considered in 21], where Tamburini showed that $\varpi\left(\mathrm{SL}_{n}(q)\right)=3$ for all $n \geq 25$ and all prime power $q$. Woldar 29] proved that all simple sporadic groups are $(2,3)$ generated, except for $\mathrm{M}_{11}, \mathrm{M}_{22}, \mathrm{M}_{23}$ and $\mathrm{McL}$, for which $\varpi(G)=5$. As proved by Lübeck and Malle [9], all simple exceptional groups are $(2,3)$-generated with the only exception of the Suzuki groups $\mathrm{Sz}\left(2^{2 m+1}\right)$, for which Suzuki himself [18] proved that $\varpi\left(\mathrm{Sz}\left(2^{2 m+1}\right)\right)=5$.

Hence, we are left to consider the finite simple classical groups. A key result for such groups is due to Liebeck and Shalev, who proved in [8] that, apart from the infinite families $\mathrm{PSp}_{4}\left(2^{m}\right)$ and $\mathrm{PSp}_{4}\left(3^{m}\right)$, all finite simple classical groups are $(2,3)$-generated with a finite number of exceptions. So, the problem of finding the exact value of $\varpi(G)$ reduces to classifying the exceptions to the Liebeck and Shalev' theorem. However, their result relies on probabilistic methods and does not provide any estimates on the number or the distribution of such exceptions. We remark that King proved in $[6$ that $\varpi(G) \neq \infty$ for all finite simple classical groups $G$, but in general the problem of computing the exact value of $\varpi(G)$ is still wide open (see 14 for a recent survey on this topic).

In this paper we consider the projective special linear groups $\operatorname{PSL}_{n}(q)$. Many authors, such as Di Martino, Macbeath Tabakov, Tamburini and Vavilov, already

2010 Mathematics Subject Classification. 20G40, $20 \mathrm{~F} 05$.

Key words and phrases. generation; linear groups. 
dealt with the problem of the $(2,3)$-generation of $\mathrm{SL}_{n}(q)$. Summarizing their results we have the following list of $(2,3)$-generated groups:

(i) $\mathrm{PSL}_{2}(q)$ if $q \neq 9$ (see [10]);

(ii) $\mathrm{SL}_{3}(q)$ if $q \neq 4$ (see [15]);

(iii) $\mathrm{SL}_{4}(q)$ if $q \neq 2$ (see [16]);

(iv) $\mathrm{SL}_{n}(q)$ if $5 \leq n \leq 11$ (see [15, 20, 19, 4, 5]);

(v) $\mathrm{SL}_{n}(q)$ if $n \geq 13$ (see [22]);

(vi) $\mathrm{SL}_{n}(q)$ if $n \geq 5$ and $q \neq 9$ is odd (see [2, 3]).

We observe that the $(2,3)$-generation of $\mathrm{SL}_{n}(q)$ clearly implies the $(2,3)$-generation of $\operatorname{PSL}_{n}(q)$.

Here, using a constructive approach as in many of the above papers and in particular the permutational method illustrated in [22, we solve the last remaining case, i.e. we prove the $(2,3)$-generation of $\mathrm{SL}_{12}(q)$, obtaining the following classification.

Theorem 1.1. The groups $\mathrm{PSL}_{2}(q)$ are $(2,3)$-generated for any prime power $q$, except when $q=9$. The groups $\mathrm{SL}_{n}(q)$ are $(2,3)$-generated for any prime power $q$ and any integer $n \geq 3$, except when $(n, q) \in\{(3,4),(4,2)\}$.

Observe that $\varpi(G)=5$ if $G \in\left\{\mathrm{PSL}_{2}(9) \cong \mathrm{Alt}(6), \mathrm{SL}_{3}(4), \mathrm{PSL}_{3}(4), \mathrm{PSL}_{4}(2) \cong\right.$ Alt(8)\}. Clearly $\mathrm{SL}_{2}(q)$ cannot be $(2, r)$-generated when $q$ is odd, as the unique involution is the central one.

Regarding the $(2,3)$-generation of the other finite classical groups, we recall that only partial results are available, mainly concerning small or high dimensions, see [12, 13, 15, 16, 23, 24, 25].

Finally, we recall that the infinite groups $\operatorname{PSL}_{n}(\mathbb{Z})$ are $(2,3)$-generated if and only if either $n=2$ or $n \geq 5$, and that the groups $\mathrm{SL}_{n}(\mathbb{Z})$ are $(2,3)$-generated if and only if $n \geq 5$ (see $22,26,27,28$ ).

\section{The $(2,3)$-Generation of $\operatorname{SL}_{12}(q)$}

Let $q=p^{a}$, where $p$ is a prime and let $\mathbb{F}_{q}$ be the field of $q$ elements. Let $V$ be a 12-dimensional $\mathbb{F}_{q}$-space, that we identify with the row vectors of $\mathbb{F}_{q}^{12}$. Let $\mathcal{C}=\left\{e_{1}, e_{2} \ldots, e_{12}\right\}$ be the canonical basis of $V$. For any element $\sigma \in \operatorname{Alt}(\mathcal{C})$, we write $g=\sigma$ to denote the permutation matrix $g \in \mathrm{SL}_{12}(q)$ corresponding to $\sigma$ with respect to $\mathcal{C}$. This allows us to consider $\operatorname{Alt}(\mathcal{C})$ as a subgroup of $\mathrm{SL}_{12}(q)$.

Now, let

$$
y=\left(e_{1}, e_{2}, e_{3}\right)\left(e_{4}, e_{5}, e_{6}\right)\left(e_{7}, e_{8}, e_{9}\right)\left(e_{10}, e_{11}, e_{12}\right)
$$

and let $x$ be the matrix, written with respect to $\mathcal{C}$, such that:

(a) $x$ swaps $e_{1}$ and $e_{8}$;

(b) $e_{2} x=-e_{2}$ and $e_{5} x=e_{5}$;

(c) $x$ swaps $e_{3 i}$ and $e_{3 i+1}$ for all $1 \leq i \leq 3$;

(d) $x$ acts on $\left\langle e_{11}, e_{12}\right\rangle$ as the matrix $\left(\begin{array}{cc}1 & 0 \\ t & -1\end{array}\right)$ with $t \in \mathbb{F}_{q}$.

Clearly $x$ and $y$ have orders, respectively, 2 and 3, and

$$
H=\langle x, y\rangle
$$

is a subgroup of $\mathrm{SL}_{12}(q)$.

First of all we prove the following.

Lemma 2.1. If $p \neq 5$, then the group $H$ contains $\operatorname{Alt}(\mathcal{C})$. 
Proof. Let $c=[x, y]=x^{-1} y^{-1} x y$ e define $\gamma$ according to the following rule:

(a) $\gamma=c^{12}$, if $p=2$;

(b) $\gamma=c^{12 p}$, if $p \equiv 1(\bmod 10)$;

(c) $\gamma=c^{24 p}$, if $p \equiv 3(\bmod 10)$;

(d) $\gamma=c^{6 p}$, if $p \equiv 7(\bmod 10)$;

(e) $\gamma=c^{18 p}$, if $p \equiv 9(\bmod 10)$.

It is easy to see that $e_{1} \gamma=-e_{3}, e_{3} \gamma=e_{5}, e_{5} \gamma=e_{4}, e_{4} \gamma=-e_{8}$ and $e_{8} \gamma=e_{1}$. Furthermore, $e_{i} \gamma=e_{i}$ for all $i \in\{2,6,7,9,10,11,12\}$. Also taking $\delta=\gamma^{y}$, we define

$$
\eta_{1}=\left(\gamma^{4} \delta^{3} \gamma^{2} \delta^{2}\right)^{2}, \quad \eta_{2}=\left(\gamma^{4} \delta^{3} \gamma^{2} \delta^{2} \gamma^{2} \delta^{2}\right)^{2}, \quad \eta_{3}=\left(\delta \gamma^{2} \delta \gamma^{2} \delta \gamma^{3} \delta^{4} \gamma^{2}\right)^{2} .
$$

Since

$$
\eta_{1}=\left(e_{2}, e_{5}\right)\left(e_{4}, e_{8}\right), \quad \eta_{2}=\left(e_{1}, e_{6}\right)\left(e_{4}, e_{9}\right), \quad \eta_{3}=\left(e_{1}, e_{3}\right)\left(e_{2}, e_{8}\right)\left(e_{4}, e_{9}\right)\left(e_{5}, e_{6}\right),
$$

we obtain that $\left\langle\eta_{1}, \eta_{2}, \eta_{3}\right\rangle=\operatorname{Alt}(\Delta)$, where $\Delta=\left\{e_{1}, e_{2}, e_{3}, e_{4}, e_{5}, e_{6}, e_{8}, e_{9}\right\} \subset \mathcal{C}$. It follows that $\langle\gamma, \delta\rangle$ contains the subgroup $\operatorname{Alt}(\Delta)$ and in particular the element $g=\left(e_{1}, e_{4}, e_{9}\right)$. Since $g^{x}=\left(e_{3}, e_{10}, e_{8}\right)$, we conclude that $H$ contains the subgroup $\left\langle\operatorname{Alt}(\Delta), g^{x}, y\right\rangle=\operatorname{Alt}(\mathcal{C})$.

The next key ingredient is the following result, which is a particular case of [22, Lemma 4.1]. As usual, $E_{i, j}$ denotes the elementary matrix having 1 at position $(i, j)$ and 0 elsewhere.

Lemma 2.2. Let $t \neq 0,2$ be such that $\mathbb{F}_{q}=\mathbb{F}_{p}(t)$. Then, the normal closure $N$ of the involution $w=I_{5}-2 E_{5,5}+t E_{5,4}$ under $\operatorname{Alt}(5)$ is $\left\langle\mathrm{SL}_{5}(q), \operatorname{diag}(-1,1,1,1,1)\right\rangle$.

We can now prove the following proposition that, combined with the known results on the $(2,3)$-generation of $\mathrm{SL}_{n}(q)$ described in the Introduction, immediately gives Theorem 1.1

Proposition 2.3. For all primes $p \neq 5$ and all integers $a \geq 1$, the groups $\mathrm{SL}_{12}\left(p^{a}\right)$ are $(2,3)$-generated.

Proof. Set $q=p^{a}$. Let $H=\langle x, y\rangle$ be as in (2), where the element $t \in \mathbb{F}_{q}$ in $x$ is chosen in such a way that $t \neq 0,2$ and $\mathbb{F}_{p}(t)=\mathbb{F}_{q}$. As already observed, $H \leq \mathrm{SL}_{12}(q)$. So, we have to prove that $\mathrm{SL}_{12}(q) \leq H$.

First, consider the element $g=\left(e_{1}, e_{8}\right)\left(e_{9}, e_{10}\right) \in \operatorname{Alt}(\mathcal{C})$. Then $w=g x$ acts on $\left\langle e_{8}, \ldots, e_{12}\right\rangle$ as the involution $I_{5}-2 E_{5,5}+t E_{5,4}$. By Lemma 2.2, we get that $\mathrm{SL}_{5}(q)$ is contained in $K=\left\langle w, \operatorname{Alt}\left(\left\{e_{8}, \ldots, e_{12}\right\}\right)\right\rangle$. It follows that $T=\left\langle K^{\prime}, \operatorname{Alt}(\mathcal{C})\right\rangle$ is $\mathrm{SL}_{12}(q)$. Since, by Lemma 2.1, $\operatorname{Alt}(\mathcal{C})$ is a subgroup of $H$ we have $T \leq H$, whence $H=\mathrm{SL}_{12}(q)$.

For sake of completeness, using the permutational method we now prove the $(2,3)$-generation of $\mathrm{SL}_{12}\left(5^{a}\right)$ for all $a \geq 1$.

Let $\tilde{y}=y$ be as in (11) and let $\tilde{x}$ be the matrix, written with respect to $\mathcal{C}$, such that:

(a) $e_{1} \tilde{x}=-e_{1}, e_{5} \tilde{x}=e_{5}$ and $e_{8} \tilde{x}=e_{8}$;

(b) $\tilde{x}$ swaps $e_{3 i}$ and $e_{3 i+1}$ for $i=2,3$;

(c) $\tilde{x}$ acts on $\left\langle e_{2}, e_{3}, e_{4}\right\rangle$ as the involution $x_{3}=\left(\begin{array}{lll}3 & 3 & 2 \\ 2 & 3 & 1 \\ 3 & 1 & 3\end{array}\right)$;

(d) $\tilde{x}$ acts on $\left\langle e_{11}, e_{12}\right\rangle$ as the matrix $\left(\begin{array}{cc}1 & 0 \\ t & -1\end{array}\right)$ with $t \in \mathbb{F}_{q}$. 
Also in this case, $\tilde{x}$ and $\tilde{y}$ have orders, respectively, 2 and 3 , and

$$
\widetilde{H}=\langle\tilde{x}, \tilde{y}\rangle
$$

is a subgroup of $\mathrm{SL}_{12}(q)$.

Lemma 2.4. The group $\widetilde{H}$ contains $\operatorname{Alt}(\mathcal{C})$.

Proof. Let $\tilde{c}=[\tilde{x}, \tilde{y}]$ and define $\tilde{\gamma}=\tilde{c}^{12}$ and $\tilde{\delta}=\tilde{\gamma}^{y^{2}}$. We firstly observe that both $\tilde{\gamma}$ and $\tilde{\delta}$ fix the decomposition $V=\left\langle e_{1}, \ldots, e_{8}\right\rangle \oplus\left\langle e_{9}\right\rangle \oplus \ldots \oplus\left\langle e_{12}\right\rangle$. Since $\tilde{\gamma} \tilde{\delta}^{2}$ and $\tilde{\gamma} \tilde{\delta} \tilde{\gamma}^{3} \tilde{\delta}^{3}$ have orders, respectively, 313 and 19531, we obtain that $K=$ $\langle\tilde{\gamma}, \tilde{\delta}\rangle$ coincides with the subgroup $\left\{\left(\begin{array}{c|c}A & 0 \\ \hline 0 & I_{4}\end{array}\right): A \in \mathrm{SL}_{8}(5)\right\} \cong \mathrm{SL}_{8}(5)$ (use, for instance, [7). In particular, $K$ contains the elements $g_{1}=\operatorname{diag}\left(1, x_{3}, I_{8}\right), g_{2}=$ $\left(e_{1}, e_{2}, e_{3}, e_{4}, e_{5}, e_{6}, e_{7}\right)$ and $g_{3}=\left(e_{6}, e_{7}, e_{8}\right)$. Now, as $g_{3}^{\tilde{y} g_{1} \tilde{x}}=\left(e_{4}, e_{8}, e_{10}\right)$, we obtain that $\widetilde{H}$ contains the subgroup $\left\langle g_{2}, g_{3}^{\tilde{y} g_{1} \tilde{x}}, \tilde{y}\right\rangle=\operatorname{Alt}(\mathcal{C})$.

Corollary 2.5. For all integers $a \geq 1$, the groups $\mathrm{SL}_{12}\left(5^{a}\right)$ are $(2,3)$-generated.

Proof. It suffices to repeat the proof of Proposition 2.3 using $\tilde{x}, \tilde{y}, \widetilde{H}$ instead of $x, y, H$, respectively, and defining $w=g \tilde{x}$, where $g=\left(e_{6}, e_{7}\right)\left(e_{9}, e_{10}\right)$.

\section{REFERENCES}

[1] M. Aschbacher and R. Guralnick, Some applications of the first cohomology group, J. Algebra 90 (1984), 446-460.

[2] L. Di Martino and N. Vavilov, (2,3)-generation of $\operatorname{SL}(n, q)$. I. Cases $n=5,6,7$, Comm. Algebra 22 (1994), 1321-1347.

[3] L. Di Martino and N. Vavilov, (2,3)-generation of SL $(n, q)$. II. Cases $n \geq 8$. Comm. Algebra 24 (1996), 487-515.

[4] E. Gencheva and Ts. Genchev, (2,3)-generation of the special linear groups of dimension 8, Mathematics and Education in Mathematics, 2015, Proceedings of the Forty Fourth Spring Conference of the Union of Bulgarian Mathematicians, SOK "Kamchia", April 2-6 (2015), 167-173.

[5] E. Gencheva, Ts. Genchev and K. Tabakov, (2,3)-generation of the special linear groups of dimensions 9, 10 and 11, arxiv.org/pdf/1412.8631v5.pdf.

[6] C.S.H. King, Generation of finite simple groups by an involution and an element of prime order, preprint.

[7] M. Liebeck, C.E. Praeger and J. Saxl, Transitive Subgroups of Primitive Permutation Groups, J. Algebra 234 (2000), 291-361.

[8] M.W. Liebeck and A. Shalev, Classical groups, probabilistic methods, and the $(2,3)$ generation problem, Ann. Math. (2) 144 (1996), 77-125.

[9] F. Lübeck and G. Malle, (2,3)-generation of exceptional groups, J. London Math. Soc. (2) 59 (1999), 109-122.

[10] A.M. Macbeath, Generators for the linear fractional groups, Proc. Simp. Pure Math. 12 (1969), 14-32.

[11] G.A. Miller, On the groups generated by two operators, Bull. AMS 7 (1901), 424-426.

[12] M.A. Pellegrini, The (2,3)-generation of the classical simple groups of dimensions 6 and 7 , Bull. Aust. Math. Soc. 93 (2016), 61-72.

[13] M.A. Pellegrini, M. Prandelli and M.C. Tamburini Bellani, The (2,3)-generation of the special unitary groups of dimension 6, J. Algebra Appl. 15 (2016) 1650171, 12 pp.

[14] M.A. Pellegrini and M.C. Tamburini, Finite simple groups of low rank: Hurwitz generation and (2,3)-generation, Int. J. Group Theory 4 (2015), 13-19.

[15] M.A. Pellegrini and M.C. Tamburini Bellani, The simple classical groups of dimension less than 6 which are (2,3)-generated, J. Algebra Appl. 14 (2015), 1550148, 15 pp.

[16] M.A. Pellegrini, M.C. Tamburini Bellani and M.A. Vsemirnov, Uniform $(2, k)$-generation of the 4-dimensional classical groups, J. Algebra, 369 (2012), 322-350.

[17] R. Steinberg, Generators for simple groups, Canad. J. Math 14 (1962), 277-283. 
[18] M. Suzuki, On a class of doubly transitive groups, Ann. of Math. (2) 75 (1962), 105-145.

[19] K. Tabakov, (2,3)-generation of the groups $\operatorname{PSL}_{7}(q)$, Proceedings of the Forty Second Spring Conference of the Union of Bulgarian Mathematicians, Borovetz, April 2-6 (2013), 260-264.

[20] K. Tabakov, and K. Tchakerian, (2,3)-generation of the groups $\mathrm{PSL}_{6}(q)$, Serdica Math J. 37 (2011), 365-370.

[21] M.C. Tamburini, Generation of certain simple groups by elements of small order, Istit. Lombardo Accad. Sci. Lett. Rend. A 121 (1987), 21-27.

[22] M.C. Tamburini, The (2,3)-generation of matrix groups over the integers, Ischia group theory 2008, 258-264, World Sci. Publ., Hackensack, NJ, 2009.

[23] M.C. Tamburini and J.S. Wilson, On the $(2,3)$-generation of some classical groups. II, J. Algebra 176 (1995), 667-680.

[24] M.C. Tamburini, J.S. Wilson and N. Gavioli, On the (2,3)-generation of some classical groups. I, J. Algebra 168 (1994), 353-370.

[25] M.C. Tamburini Bellani and M. Vsemirnov, Hurwitz generation of $\operatorname{PSp}_{6}(q)$, Comm. Algebra 43 (2015), 4159-4169.

[26] M.A. Vsemirnov, Is the group SL(6,Z) (2,3)-generated?, Zap. Nauchn. Sem. S.-Peterburg. Otdel. Mat. Inst. Steklov. (POMI) 330 (2006), Vopr. Teor. Predst. Algebr. i Grupp. 13, 101-130, 272; English translation in J. Math. Sci. (N. Y.) 140 (2007), 660-675.

[27] M.A. Vsemirnov, On the (2,3)-generation of matrix groups over the ring of integers, Algebra $i$ Analiz 19 (2007), 22-58; English translation in St. Petersburg Math. J. 19 (2008), 883-910.

[28] M.A. Vsemirnov, On the (2,3)-generation of small rank matrix groups over integers, Quaderni del Seminario Matematico di Brescia 30 (2008), 1-15.

[29] A.J. Woldar, On Hurwitz generation and genus actions of sporadic groups, Illinois Math. J. (3) 33 (1989), 416-437.

Dipartimento di Matematica e Fisica, Università Cattolica del Sacro Cuore, Via Musei 41, 25121 Brescia, Italy

E-mail address: marcoantonio.pellegrini@unicatt.it 